\title{
(Semi)classical limits of Schrödinger-Poisson systems via Wigner transforms
}

\author{
Norbert J. Mauser
}

\begin{abstract}
We deal with classical and "semiclassical limits", i.e. vanishing Planck constant $\hbar \simeq \epsilon \rightarrow 0$, eventually combined with a homogenization limit of a crystal lattice, of a class of "weakly nonlinear" NLS. The Schrödinger-Poisson (S-P) system for the wave functions $\left\{\psi_{j}^{\epsilon}(t, x)\right\}$ is transformed to the WignerPoisson (W-P) equation for a "phase space function" $f^{\epsilon}(t, x, \xi)$, the Wigner function. The weak limit of $f^{\epsilon}(t, x, \xi)$, as $\epsilon$ tends to 0 , is called the "Wigner measure" $f(t, x, \xi)$ (also called "semiclassical measure" by P. Gérard).

The mathematically rigorous classical limit from S-P to the Vlasov-Poisson (V-P) system has been solved first in 1993 by P.L. Lions and T. Paul in [21] and, independently, by P.A. Markowich and N.J. Mauser in [23]. There the case of the so called "completely mixed state", i.e. $j=1,2, \ldots, \infty$, was considered where strong additional assumptions can be posed on the initial data.

For the so called "pure state" case where only one (or a finite number) of wave functions $\left\{\psi_{j}^{\epsilon}(t, x)\right\}$ is considered, recently P. Zhang, Y. Zheng and N.J. Mauser [33] have given the limit from S-P to V-P in one space dimension for a very weak class of measure valued solutions of V-P that are not unique.

For the setting in a crystal, as it occurs in semiconductor modeling, we consider Schrödinger equations with an additional periodic potential. This allows for the use of the concept of "energy bands", Bloch decomposition of $L^{2}$ etc. On the level of the Wigner transform the Wigner function $f^{\epsilon}(t, x, \xi)$ is replaced by the "Wigner series" $f^{\epsilon}(t, x, k)$, where the "kinetic variable" $k$ lives on the torus ("Brioullin zone") instead of the whole space.

Recently P. Bechouche, N.J. Mauser and F. Poupaud [7] have given the rigorous "semiclassical" limit from S-P in a crystal to the "semiclassical equations", i.e. the "semiconductor V-P system", with the assumption of the initial data to be concentrated in isolated bands.
\end{abstract}

Support by the Austrian START project "Nonlinear Schrödinger and quantum Boltzmann equations" is acknowledged.

MSC 2000: 35L65, 35Q55, 81Q05, 81Q20.

Keywords : nonlinear Schrödinger equations, Schrödinger-Poisson, Vlasov-Poisson, classical limit, semiclassical equations, Wigner measure, Wigner transform, Wigner Bloch series. 


\section{Introduction}

We consider the Schrödinger-Poisson (S-P) system as a special weakly nonlinear "one-particle" Schrödinger equation :

$$
\begin{aligned}
i \epsilon \partial_{t} \psi_{j}^{\epsilon} & =-\frac{\epsilon^{2}}{2} \Delta \psi_{j}^{\epsilon}+V^{\epsilon} \psi_{j}^{\epsilon}, & & x \in \mathbb{R}^{n}, \quad t>0, \\
\left.\psi_{j}^{\epsilon}\right|_{t=0} & =\phi_{j}^{\epsilon}, & & x \in \mathbb{R}^{n}, \quad j \in \mathbb{N} .
\end{aligned}
$$

The "asymptotic parameter" $\epsilon>0$ is the scaled Planck constant $\hbar$. The index $j \in \mathbb{N}$ means that we consider a set of possibly infinite many Schrödinger equations, which is called a "mixed state" where the particle density $\rho^{\epsilon}(t, x)$ and the "current density" $J^{\epsilon}(t, x)$ are given by weigthed sums as

$$
\rho^{\epsilon}(t, x)=\sum_{j=1}^{\infty} \lambda_{j}^{\epsilon}\left|\psi_{j}^{\epsilon}(t, x)\right|^{2}, \quad J^{\epsilon}(x, t):=\epsilon \sum_{j \in \mathbb{N}} \lambda_{j}^{\epsilon} \operatorname{Im}\left(\nabla \psi_{j}^{\epsilon}(t, x) \cdot \overline{\psi_{j}^{\epsilon}(t, x)}\right)
$$

where the coefficients $\lambda_{j}^{\epsilon} \geq 0$ are the "occupation probabilities" of $L^{2}\left(\mathbb{R}^{n}\right)$-orthonormalized initial states $\phi_{j}^{\epsilon}(x)$. These $\lambda_{j}^{\epsilon}$ are constant in the time evolution, but depend in general on $\epsilon$, which is crucial for the classical limit.

The potential $V^{\epsilon}$ in (1.1) is a multiplication operator that models the interaction of the "particle" with the rest of the world. The (Coulomb) interaction among electrons and with a positively charged background ("doping profile" $b(x) \geq 0$ ) is modeled as

$$
V^{\epsilon}=-\tilde{V} *\left(\rho^{\epsilon}-b\right),
$$

where $\tilde{V}$ represents the two body interaction potential that we consider. If $\tilde{V}$ is the Green's function of the Laplace operator then the integral representation (1.3) can be rewritten as the Poisson equation :

$$
\Delta V^{\epsilon}=-C\left(\rho^{\epsilon}-b\right)
$$

Note that only in the 3 -d case the Coulomb interaction of point particles and the Green's function of Laplace coincide. The use of the Poisson equation in lower dimensions means that we no longer consider point particles.

For this case where $V^{\epsilon}$ is the Coulomb potential (1.4) it is known from [12], [13] that (1.1) has a global smooth solution $\left(\psi_{j}^{\epsilon}(t, x)\right)$ such that the mass is conserved and the kinetic energy is uniformly (in $\epsilon$ ) bounded :

$$
\sum_{j=1}^{\infty} \lambda_{j}^{\epsilon}\left\|\psi_{j}^{\epsilon}(t, \cdot)\right\|_{L^{2}}^{2}+\epsilon^{2} \sum_{j=1}^{\infty} \lambda_{j}^{\epsilon}\left\|\nabla \psi_{j}^{\epsilon}\right\|_{L^{2}}^{2}<\infty
$$

Despite its wide use the rigorous derivation of the time-dependent S-P equation has been an open problem for decades and only recently two completely different ways of obtaining the S-P system have been successfull :

The most fundamental way to derive S-P is by a weak coupling limit of the $N$ particle Schrödinger Coulomb system which has been done in a series of papers by C. Bardos, F. Golse, N.J. Mauser and L. Erdös, H.T. Yau [3], [16], [2]. 
For an improvement of this very crude approximation of the linear $N$ particle problem by a nonlinear "mean field" one-particle equation, based on a molecular chaos ansatz (Hartree ansatz) for $N$ interacting electrons, the inclusion of the Pauli principle for fermions would be essential. However, the attempts to incorporate the resulting "exchange interaction" as a local term in the time-dependent S-P model, are heuristic (cf [28]) up to now. Steps in this direction are the recent progress on the derivation of the time dependent Hartree-Fock (HF) equation [4], [5] and the derivation of the " $X \alpha$ " potential as a local approximation of the HF exchange potential [10], [11].

Another direction of extending the S-P model is to incorporate relativistic corrections, at least at first order, which yields the Pauli equation where spin and magnetic field are taken into account. A consistent first order semi-nonrelativistic timedependent model is given by the self-consistent "Pauli-Poiswell" equation which was introduced in [26]. The classical limit of this system seems doable analogous the the S-P case with the methods and assumptions of [21], with appropriate "magnetic Lieb-Thirring type estimates".

The rigorous derivation of the S-P system as the non-relativistic limit of the Dirac-Maxwell and the Klein Gordon Maxwell system has been given by P. Bechouche, N.J. Mauser, S. Selberg and N. Masmoudi, K. Nakanishi [8], [9] , [27].

For modeling electrons in a semiconductor [25] a key modification of the S-P equation is to take into account the additional periodic "crystal potential". The starting point is hence a a mixed state Schrödinger equation with a periodic potential due to the ions of a crystal lattice and a nonperiodic potential due to the selfconsistent Coulomb interaction among the electrons, i.e.

$$
i \hbar \partial_{t} \psi_{j}(t, x)=\left(-\frac{\hbar^{2}}{2 m} \Delta+V_{p e r}(x)+V(t, x)\right) \psi_{j}(t, x), \quad j \in \mathbb{N},
$$

with a given periodic potential $V_{p e r}(x)$ and a nonperiodic potential $V(t, x)$.

On a semiclassical level of description [1], the Schrödinger equation (1.6) is replaced by the semiclassical Liouville equation for the phase space (i.e. position-wave vector space) density $f=f(t, x, k)$, where $k \in B$, the bounded Brillouin zone (the torus). Under the assumption that the electron "stays in the $m$-th (energy) band $E_{m}(k)$ " the semiclassical Liouville equation reads [1]

$$
\partial_{t} f+\frac{1}{\hbar} \nabla_{k} E_{m}(k) \cdot \nabla_{x} f-\frac{1}{\hbar} \nabla_{x} V(t, x) \cdot \nabla_{k} f=0, \quad x \in \mathbb{R}, k \in B, t \in \mathbb{R}
$$

subject to a periodic boundary condition in $k$ and an initial condition $f(t=0)=f_{I}$. The position density $\rho$ and the current density $J$ are now computed as

$$
\rho(t, x)=\frac{1}{4 \pi^{3}} \int_{B} f d k, \quad J(t, x)=\frac{1}{4 \pi^{3}} \int_{B} \frac{1}{\hbar} \nabla_{k} E_{m} f d k
$$

The self-consistent potential $V(t, x)$ is again calculated by solution of the Poisson equation (1.4) with $\rho$ given by (1.8).

The transition from the quantum model (1.6), (1.2), (1.4) to the semiclassical model (1.7), (1.8), (1.4) has been made rigorous in [7] where a new variant of Wigner 
transforms, the Wigner-Bloch series were introduced. The reader is referred to [24], [7] for a comprehensive presentation and precise results.

By "classical limit" we mean the transition from quantum mechanics to classical mechanics for vanishing Planck constant $\epsilon \rightarrow 0$; without keeping any "semiclassical" corrections. Note that we avoid the frequently used term "semiclassical limit" for $\epsilon \rightarrow 0$; in particular since in solid state physics the term "semiclassical equation" is reserved for (1.7) as a "crystal" version of the Vlasov equation. The limit from a Schrödinger equation with a periodic potential, which yields the "semiclassical equations" as a simultaneous classical limit plus homogenization limit of the periodic structure, deserves the name "semiclassical limit" and in fact quantum corrections are contained in the limit via the "energy bands" ( $\epsilon=\hbar$ is even explicitly contained in $(1.7))$.

For the purpose of "semiclassical approximations" of quantum mechanics, E. Wigner ([31]) introduced the "Wigner transform" from "physical space" to "phase space" :

$$
f^{\epsilon}(t, x, \xi)=\frac{1}{(2 \pi)^{n}} \int_{\mathbb{R}^{n}} e^{-i \xi y} z^{\epsilon}\left(t, x+\frac{\epsilon y}{2}, x-\frac{\epsilon y}{2}\right) d y,
$$

where $z^{\epsilon}(t, r, s)$ is the so-called mixed state density matrix defined by

$$
z^{\epsilon}(t, r, s)=\sum_{j=1}^{\infty} \lambda_{j}^{\epsilon} \psi_{j}^{\epsilon}(t, r) \overline{\psi_{j}^{\epsilon}(t, s)}, \quad r, s \in \mathbb{R}^{n} .
$$

We use "overhead bar" for complex conjugate. Note that $z^{\epsilon}(t, r, s)$ is the integral kernel of the "density operator" $\hat{\rho}^{\epsilon}$ in $L^{2}$, which is trace class with $\operatorname{Tr}\left(\hat{\rho}^{\epsilon}\right)=$ $\sum_{j=1}^{\infty} \lambda_{j}^{\epsilon}=1$.

A direct calculation by applying the transform (1.9) to the Schrödinger equation (1.1) (see e.g. [21], [23]) shows that $f^{\epsilon}(t, x, \xi)$ satisfies the so-called Wigner equation

$$
\begin{aligned}
& \partial_{t} f^{\epsilon}+\xi \nabla_{x} f^{\epsilon}+\theta^{\epsilon}\left[V^{\epsilon}\right] f^{\epsilon}=0, \quad x, \xi \in \mathbb{R}^{n}, \quad t>0, \\
& \left.f^{\epsilon}\right|_{t=0}=f_{I}^{\epsilon}(x, \xi),
\end{aligned}
$$

where $\theta^{\epsilon}\left[V^{\epsilon}\right] f^{\epsilon}$ is the pseudo-differential operator (local in $x$ and nonlocal in $\xi$ )

$$
\theta^{\epsilon}\left[V^{\epsilon}\right] f^{\epsilon}=\frac{i}{(2 \pi)^{n}} \iint_{\mathbb{R}_{\eta, y}^{2 n}} \frac{V^{\epsilon}\left(x+\frac{\epsilon y}{2}\right)-V^{\epsilon}\left(x-\frac{\epsilon y}{2}\right)}{\epsilon} f^{\epsilon}(t, x, \eta) e^{-i(\xi-\eta) y} d \eta d y,
$$

associated to the potential $V^{\epsilon}$ and the initial datum $f_{I}^{\epsilon}(x, \xi)$ is the Wigner transform of the initial density matrix $z_{I}^{\epsilon}(r, s)=\sum_{j=1}^{\infty} \lambda_{j}^{\epsilon} \phi_{j}^{\epsilon}(r) \overline{\phi_{j}^{\epsilon}(s)}$.

The macroscopic densities $\rho^{\epsilon}(t, x)$ and the current density $J^{\epsilon}(t, x)$ are now given as the zero order and the first order moment, respectively, in the kinetic variable $\xi$, i.e. by a linear expression in $f^{\epsilon}$ due to the quadratic nature of the Wigner transform, in complete analogy to classical and "semiclassical" (cf (1.8)) physics.

$$
\rho^{\epsilon}(t, x)=\int_{\mathbb{R}^{n}} f^{\epsilon}(t, x, \xi) d \xi \geq 0, \quad \quad J^{\epsilon}(t, x)=\int_{\mathbb{R}^{n}} \xi f^{\epsilon}(t, x, \xi) d \xi
$$


The global existence and uniqueness of classical solution for the Wigner-Poisson (W-P) system, i.e (1.11) coupled to (1.4) using (1.13) was proven (in 3-d) in [12].

An important advantage of using the Wigner transform lies in the structural similarity of the quantum Wigner equation (1.11) with the classical Vlasov equation and the fact that the role of the moments is the same (cf (1.13)). Formally passing $\epsilon \rightarrow 0$ in (1.11), the pseudodifferential operator (1.12) becomes $\nabla_{x} V \cdot \nabla_{\xi} f$. Hence formally the limit of the W-P equation $(1.11),(1.4)$ is given by the $\mathrm{V}-\mathrm{P}$ equation, where :

$$
\begin{aligned}
& \partial_{t} f+\xi \cdot \nabla_{x} f-\nabla_{x} V \cdot \nabla_{\xi} f=0, \quad x, \xi \in \mathbb{R}^{n}, \quad t>0 \\
& \Delta V=-C(\rho(t, x)-b(x)) \\
& \left.f\right|_{t=0}=f_{I}
\end{aligned}
$$

where $f(t, x, \xi), f_{I}(x, \xi), V(t, x)$ are the formal limits of $f^{\epsilon}(t, x, \xi), f_{I}^{\epsilon}(x, \xi), V^{\epsilon}(t, x)$ and $\rho(t, x)=\int_{\mathbb{R}^{n}} f(t, x, \xi) d \xi$.

There are several serious problems in justifying this formal limit which can a priori only be some sort of weak limit since the Wigner function inherits the $\epsilon$ oscillatory behaviour from the wave functions. The first rigorous proof was not given before 1993 by P.L. Lions and T. Paul [21] and by P.A. Markowich and N.J. Mauser [23]. Note that the Wigner function $f^{\epsilon}$ is in general real, but has also negative values, whereas the limit $f$ is a "true", nonnegative distribution function. In [21] and [23], independently, the method of using a smoothed Wigner function, the "Husimi function" (which is closely related to the "coherent states"), was introduced to overcome this problem.

By the special structure of the "mixed state" Schrödinger equations (1.1), the natural assumption for the initial data of (1.1), i.e. $\lambda_{j}^{\epsilon}$ and $\phi_{j}^{\epsilon}$ should be $\phi_{j}^{\epsilon} \in H^{s}(\mathbb{R})$ with $s$ large and the conservation of mass : $\sum_{j=1}^{\infty} \lambda_{j}^{\epsilon}$ and the bound on the kinetic energy : $\epsilon^{2} \sum_{j=1}^{\infty} \lambda_{j}^{\epsilon}\left\|\nabla \phi_{j}^{\epsilon}\right\|_{L^{2}}^{2}<\infty$. Then (1.5) holds for $t>0$ and thus by [21] or [17] it follows that $f(t, \cdot, \cdot)$ is in fact a Wigner measure.

\section{Results on classical limits}

The problem we deal with is to rigorously justify the limit for the Coulomb case of the weak "self consistent" nonlinearity (1.3), i.e. from S-P (1.11) to V-P (1.14), (1.15).

In the results [21], [23] this problem was solved for a "really mixed state" where infinitely many wave functions contribute in a very particular dependence on the Planck constant $\epsilon$, as stated in the following assumption

$$
\frac{1}{\epsilon^{3}} \sum_{j=1}^{\infty}\left(\lambda_{j}^{\epsilon}\right)^{2} \leq C
$$

Note that the 1.h.s. of (2.16) corresponds to the trace of the square of the density operator associated to (1.10), i.e. $\sum_{j=1}^{\infty}\left(\lambda_{j}^{\epsilon}\right)^{2}=\operatorname{Tr}\left(\left(\hat{\rho}^{\epsilon}\right)^{2}\right)$. We see from (1.9) that the r.h.s of (2.16) is the uniform (in $\epsilon$ ) bound in $L^{2}\left(\mathbb{R}_{x}^{n} \times \mathbb{R}_{\xi}^{n}\right)$ of the Wigner functions 
$f^{\epsilon}$ (up to a factor $\left.(2 \pi)^{3}\right)$. However, the uniform bound on $f^{\epsilon}$ of Proposition 1 below does not need the additional assumption (2.16), but only the uniform $L^{2}$ bounds of the wave functions $\psi^{\epsilon}$.

In [33] the classical limit of the one dimensional S-P to the V-P system for general initial data; in particular the "pure state case", without assumption (2.16), was given. The trick in the pure state case is to change the concept of solutions of $\mathrm{V}$-P such that jumps in $\nabla V$ are allowed and $\mathcal{A}^{\prime}$ convergence of the Wigner measure $f$ is enough. The price to pay is loss of uniqueness in the $\mathrm{V}-\mathrm{P}$ system.

The space of finite nonnegative Radon measures is denoted by $\mathcal{M}^{+}$. For the study of the convergence of the Wigner function $f^{\varepsilon}$, the following space $\mathcal{A}$ has been introduced in [21] which is a separable Banach algebra of test functions containing $S\left(\mathbb{R}_{x}^{n} \times \mathbb{R}_{\xi}^{n}\right):$

$$
\begin{array}{r}
\mathcal{A}=\left\{\varphi \in C_{0}\left(\mathbb{R}_{x}^{n} \times \mathbb{R}_{\xi}^{n} \mid\left(\mathcal{F}_{\xi} \varphi\right)(x, \eta) \in L^{1}\left(\mathbb{R}_{\eta}^{n} ; C_{0}\left(\mathbb{R}_{x}^{n}\right)\right)\right\}\right. \\
\left\|\mathcal{F}_{\xi} \varphi\right\|_{L_{\eta}^{1}\left(C_{x}\right)}=\int_{\mathbb{R}_{\eta}^{m}} \sup _{x}\left|\mathcal{F}_{\xi} \varphi\right|(x, \eta) d \eta
\end{array}
$$

This space immediately allows for a uniform estimate on the Wigner function :

Proposition 1 Let the sequence $\psi^{\varepsilon}$ be uniformly bounded in $L^{2}$. Then the sequence of Wigner transforms $f^{\varepsilon}$ of $\psi^{\varepsilon}$ is uniformly bounded in $\mathcal{A}^{\prime}$.

Hence $f^{\varepsilon}$ converges, possibly after extracting a subsequence, to a distribution $\mu$ in $\mathcal{A}^{\prime}$ weak $-*$. Note that $\mu$ is in general not unique, since it may depend on the selection of a particular subsequence. It can be proven [21], [17] that $\mu$ is in fact a nonnegative measure, thus justifying the term 'Wigner measure' and the identfication $\mu=f$, where $f$ stands for a solution of a Vlasov type equation.

For the "pure state" result below this non-uniqueness of the limiting Wigner measure corresponds to the non-uniqueness of the kind of solutions of V-P introduced in [34] and used in [33]. See [22] for a construction of non-unique solutions to 1-d V-P (in a setting which is periodic in $x$, however) where different "regularization schemes" give different limits (cf also the remarks in [35]). Hence in this formulation the classical limit of the quantum problem does not provide a selection criterion among the non-unique measure-valued solutions of the V-P equation in the sense of Section 2.2.

A word of caution, however, should be said on the physical interpretation : terms like "concentration in a point charge" for the singularities are somewhat misleading in a low-dimensional V-P model which actually do not model point charges but infinite planes (1-d model) or straight wires (2-d model) since we have to assume translational invariance in 2 or 1 directions for obtaining the Poisson equation.

The physical interpretation, why in the techniques of [21] and [23] (that apply also to the 2- and 3-d case where no pure state result is known) the mixed state is so different from the pure state is not obvious. In some sense a mixed state obeying (2.16), i.e. a density operator which has the property that the trace norm of its square tends to zero with the (third power of the) Planck constant, is closer to classical mechanics than a pure state, which is reflected in a uniformly $L^{2}\left(\mathbb{R}_{x}^{n} \times \mathbb{R}_{\xi}^{n}\right)$ bound for the Wigner function and the admissible initial Wigner measures. 


\subsection{S-P to V-P "in vacuum" for mixed states}

The results of [21] and [23] yield the limit from S-P to V-P only for the mixed state case. In [21], in addition, an exhaustive list of results, including the linear case, is given. We just sketch the results on the weakly nonlinear case.

Let $\psi^{\varepsilon}(t, x)$ be the solution of the Schrödinger equation (1.1) and $f^{\epsilon}(t, x)$ and $f(t, x)$ its Wigner function and a Wigner measure of the sequence $\psi^{\varepsilon}$.

Theorem 1.a Let $V \mid \epsilon=\tilde{V} * \rho^{\varepsilon}, \tilde{V}=\tilde{V}^{+}+\tilde{V}^{-}$and $\rho^{\varepsilon}=\left|\psi^{\varepsilon}(t, x)\right|$. Suppose that $\tilde{V}^{-} \geq-C, \tilde{V} \in C^{1}\left(\mathbb{R}^{n}\right), \nabla \tilde{V} \in C_{b}\left(\mathbb{R}^{n}\right), \operatorname{Tr}\left(\frac{\varepsilon^{2}}{2} \Delta \hat{\rho}_{I}^{\varepsilon}\right) \leq C$, and that

$$
\iint_{\mathbb{R}^{2 n}} \tilde{V}^{+}(x-y) \rho_{I}^{\varepsilon}(x) \rho_{I}^{\varepsilon}(y) d x d y \leq C, \quad \int_{\mathbb{R}^{n}}|x|^{2} \rho_{I}^{\varepsilon}(x) d x \leq C,
$$

where $\rho_{I}^{\varepsilon}=\left.\rho^{\varepsilon}\right|_{t=0}$, $\hat{\rho}_{I}^{\varepsilon}$ is the density operator in $L^{2}$. Then we have

- $f^{\varepsilon} \rightarrow f$ weak-* in $\mathcal{A}^{\prime}$ for $f \in C_{b}\left(\mathbb{R}_{t}, \mathcal{M} w-*\right)$ uniformly on all compact subsets in $\mathbb{R}_{t}$ and $f$ is a solution in $\mathcal{D}^{\prime}$ of the following nonlinear Vlasov equation

$$
\begin{array}{r}
\partial_{t} f+\operatorname{div}_{x}(\xi f)-\operatorname{div}_{\xi}\left(\nabla_{x} V^{0}(x) f\right)=0 \quad \text { with initial data }\left.\quad f\right|_{t=0}=f_{I}^{0} \\
\text { where } \quad V^{0}=\tilde{V} * \rho^{0} \quad \text { and } \quad \rho^{0}=\int_{\mathbb{R}_{\xi}^{n}} d f(\cdot, \cdot, \xi)
\end{array}
$$

Corollary 1.a If $\tilde{V} \in C^{1,1}\left(\mathbb{R}^{n}\right)$, then the above $f$ is given by the unique Hamiltonian flow of $f_{I}:(x, \xi) \rightarrow(x(t), \xi(t))$ with $\dot{x}=\xi, \dot{\xi}=-\nabla V^{0}(x), x(0)=x, \xi(0)=\xi$.

Remark ! In general, we can relax the $C^{1}$ regularity assumption on $V$ or $\tilde{V}$, but then we have to ask more properties on the initial data that are possible only for the mixed state, namely (2.16) which guarantee a better convergence of the Wigner function than in $\mathcal{A}^{\prime}$, namely weakly in $L^{2}$, in order to pass to the limit in the pseudodifferential operator (1.12) that contains the crucial product term.

Theorem 1.b Let $V^{\epsilon}=\tilde{V} * \rho^{\varepsilon}, \tilde{V}=\tilde{V}^{+}+\tilde{V}^{-}$and $\rho^{\varepsilon}=\left|\psi^{\varepsilon}(t, x)\right|$. Suppose that

$$
\begin{gathered}
\tilde{V}^{-} \in L^{\frac{n+4}{4}, \infty}\left(\mathbb{R}^{n}\right)+L^{\infty}\left(\mathbb{R}^{n}\right) \quad n \leq 3, \\
\nabla \tilde{V} \in L^{\frac{2 n+8}{n+8}}\left(\mathbb{R}^{n}\right)+L^{q}\left(\mathbb{R}^{n}\right) \quad \text { with } \frac{2 n+8}{n+8}<q<\infty, \\
\operatorname{Tr}\left(\frac{\varepsilon^{2}}{2} \Delta \hat{\rho}_{I}^{\varepsilon}\right) \leq C, \quad \iint_{\mathbb{R}^{2 n}} \tilde{V}^{+}(x-y) \rho_{I}^{\varepsilon}(x) \rho_{I}^{\varepsilon}(y) d x d y \leq C,
\end{gathered}
$$

where $\rho_{I}^{\varepsilon}=\left.\rho^{\varepsilon}\right|_{t=0}$, is the initial density and $\hat{\rho}_{I}^{\varepsilon}$ is the initial density operator in $L^{2}$. Assume initially a mixed state where (2.16) holds (which is behind (87) in [21]!

Then we have the convergence result :

- $f^{\varepsilon} \rightarrow f$ in $L^{\infty}\left((0, T) ; L^{2}\left(\mathbb{R}_{x}^{n} \times \mathbb{R}_{\xi}^{n}\right)\right.$ weak-* (and in $C\left([0, T] ; \mathcal{M}^{+} w-*\right), \forall T \in$ $(0, \infty)$, and $f \in C_{b}\left(\mathbb{R}_{t}, \mathcal{M} w-*\right) \cap L^{\infty}\left(\mathbb{R}_{t} ; L^{1} \cap L^{2}\left(\mathbb{R}_{x}^{n} \times \mathbb{R}_{\xi}^{n}\right)\right.$ is a solution of the nonlinear Vlasov equation (2.18),(2.19).

Remark 2.1 In the $S$-P case we have : $n=3, \tilde{V}=1 /|x|$ and the assumptions (2.20) and (2.21) are satisfied with $\tilde{V}^{-}=0, \nabla \tilde{V} \in L^{3 / 2, \infty}(\mathbb{R})$ and $\frac{2 n+8}{n+8}=\frac{14}{11}<\frac{3}{2}$. 


\subsection{S-P to V-P "in vacuum" in 1-d for pure states}

Before the presentation of 1-d pure state result of [33], let us first recall some related existence results for V-P, i.e. (1.14), (1.15) with $b=0$ and $\tilde{V}$ being the Coulomb potential. Note that we consider a one dimensional model by assuming translational invariance in two space dimensions, such that in (1.3) $\tilde{V}=1 /|x|$.

- For $n=3$, under the assumption that there exists some $p_{0}>3$ such that

$$
f_{I}(x, \xi) \in L^{1} \cap L^{\infty}\left(\mathbb{R}^{6}\right), \quad \int_{\mathbb{R}^{6}}|\xi|^{p} f_{I} d x d \xi<\infty, \quad \text { for all } \quad 1 \leq p<p_{0},
$$

In [20] (see also [29] and [30]) it was proved that V-P has a global distributional solution which satisfies (2.22) for all time; under the weaker assumption that

$$
\iint_{\mathbb{R}^{6}}\left(1+|\xi|^{2}+\left|\ln f_{I}\right|\right) f_{I} d x d \xi<\infty
$$

In [15] the global existence of renormalized solutions of $\mathrm{V}-\mathrm{P}$ was proved. When $f_{I}(x, \xi)$ is a nonnegative Radon measure, the global existence of weak solutions of $\mathrm{V}-\mathrm{P}$ is open.

- For $n=1$, however, i.e. in one dimension and if $f_{I}(x, \xi)$ satisfies

$$
\iint_{\mathbb{R}^{2}} e^{\alpha|\xi|} f_{I}(x, \xi) d x d \xi \leq C_{\alpha}, \quad \forall \alpha \geq 0
$$

the global existence of weak solutions of $\mathrm{V}-\mathrm{P}$ was proved in [34] in the following sense:

Definition 2.1 A pair $(E(t, x), f(t, x, \xi)))$ of a function and a finite nonnegative Radon measure is called a weak solution of $V$-P, i.e. of (1.14), (1.15), if for any $T>0$, there hold

1) $E(t, x) \in\left(B V \cap L^{\infty}\right)\left(\Omega_{T}\right) ;\left(\Omega_{T}:=[0, T] \times \mathbb{R}^{1}\right)$

2) $f(t, x, \xi) \in L^{\infty}\left(\mathbb{R}^{+}, \mathcal{M}^{+}\left(\mathbb{R}^{2}\right)\right)$;

3) $\int_{\mathbb{R}} \phi(t, x, \xi) f(t, x, \xi) d \xi=\partial_{x} g_{\phi}$ as measures for some $g_{\phi}$ in $B V\left(\Omega_{T}\right)$, for all $\phi(t, x, \xi)$ in $C_{c}^{\infty}\left(\Omega_{T} \times \mathbb{R}\right)$;

4) $E(t, x)=\int_{-\infty}^{x}\left(b(y)-\int_{\mathbb{R}} f(t, y, \xi) d \xi\right) d y$, a. e.;

5) $\forall \phi \in C_{c}^{\infty}\left((0, T) \times \mathbb{R}^{2}\right)$,

$$
\int_{0}^{T} \iint_{\mathbb{R}^{2}}\left(\phi_{t} f+\phi_{x} \xi f\right) d x d t-\int_{0}^{T} \int_{\mathbb{R}} \bar{E} \int_{\mathbb{R}} \phi_{\xi} f(d \xi) d x d t=0
$$

5b) Here the term $\bar{E}(t, x)$ in (2.25) in the above definition is the Vol'pert's symmetric average:

$\bar{E}(t, x)= \begin{cases}E(t, x), & \text { if } E \text { is approximately continuous at }(t, x), \\ \frac{1}{2}\left(E_{l}(t, x)+E_{r}(t, x)\right) & \text { if } E \text { has a jump at }(t, x),\end{cases}$

where $E_{l}(t, x)$ and $E_{r}(t, x)$ denote respectively the left and right limits of $E(t, x)$ at a discontinuity line at $(t, x)$.

6) $f \in C^{0,1}\left([0, T), H_{l o c}^{-L}\left(\mathbb{R}^{2}\right)\right.$ for some $L>0$ and $f(0, x, \xi)=f_{I}(x, \xi)$ in $H_{l o c}^{-L}\left(\mathbb{R}^{2}\right)$. 
Note that overhead bars are used to denotes complex conjugate, too, we hope it does not cause confusion with the one term $\bar{E}$. A special and very useful property of $\bar{E}$ is : if $E(t, x) \in\left(B V \cap L^{\infty}\right)\left(\mathbb{R}^{2}\right)$, then $E^{2}(t, x) \in B V\left(\mathbb{R}^{2}\right)$ and

$$
\nabla E^{2}=2 \bar{E} \nabla E
$$

in the sense of measures.

The definition 2.1 of weak solutions of V-P is used in the sequel. More precisely, when the space dimension $n=1$, under appropriate assumption on $\phi_{j}^{\epsilon}(x)$, we can show that $f^{\epsilon}(t, x, \xi)$ tend to a weak solution of (1.14), (1.15) as $\epsilon \rightarrow 0$.

In the following, we let $\psi^{\epsilon}(t, x)$ be the solution of the 1-d S-P equation

$$
\begin{aligned}
& i \epsilon \partial_{t} \psi^{\epsilon}=-\frac{\epsilon^{2}}{2} \partial_{x}^{2} \psi^{\epsilon}+V^{\epsilon} \psi^{\epsilon}, \quad \partial_{x}^{2} V^{\epsilon}=b^{\epsilon}(x)-\left|\psi^{\epsilon}\right|^{2}, \quad x \in \mathbb{R}, \quad t \geq 0, \\
& \left.\psi^{\epsilon}\right|_{t=0}=\psi_{0}^{\epsilon}
\end{aligned}
$$

where $b^{\epsilon}(x) \geq 0$ and $\psi_{0}^{\epsilon}$ will be specified later in Theorem 2.1. We assume $V^{\epsilon}$ and $V_{x}^{\epsilon}$ vanish as $x \rightarrow-\infty$.

We use the V-P system (1.14), (1.15) in the form written directly in the electric field $E=-\partial_{x} V$

$$
\begin{aligned}
& \partial_{t} f+\xi \partial_{x} f-E \partial_{\xi} f=0, \quad \partial_{x} E=b(x)-\int_{\mathbb{R}} f d \xi, \quad x \in \mathbb{R}, \quad t \geq 0, \\
& \left.f\right|_{t=0}=f_{I}
\end{aligned}
$$

We also assume that $E$ vanishes as $x \rightarrow-\infty$.

We note that it is convenient and sharp to use the space $\mathcal{A}^{\prime}$ to consider the weak convergence of $f^{\epsilon}(t, x, \xi)$. Let us add that $\mathcal{A}^{\prime}$ can be embedded in $H^{-s}\left(\mathbb{R}^{n}\right)$ with $s>\frac{n}{2}$.

Then the result on the "pure state case" is :

Theorem 2.1 Let $\varphi_{\epsilon}(x)$ be uniformly bounded in $L^{2}(\mathbb{R}), b(x) \in\left(L^{1} \cap L^{2}\right)(\mathbb{R})$, $\psi_{0}^{\epsilon}(x)=j_{\epsilon} * \varphi_{\epsilon}(x), b^{\epsilon}(x)=b * j_{\epsilon}(x)$ where $j_{\epsilon}(x)$ denotes the standard Friedrichs' mollifier, and $f^{\epsilon}(t, x, \xi)$ be the Wigner transform of the solution $\psi^{\epsilon}(t, x)$ of (2.28).

Then there is a nonnegative bounded Radon measure $f(t, x, \xi)$ such that

$$
f^{\epsilon}(t, x, \xi) \rightarrow f(t, x, \xi) \text { in } C\left([0, \infty), \mathcal{A}^{\prime}\right)
$$

and the Wigner measure $f(t, x, \xi)$ is a weak solution of (2.29) in the sense of Definition 2.1 with $f_{I}$ determined by $\psi_{0}^{\epsilon}(x)$ and an $L>\frac{3}{2}$.

Remark 2.2 1) Obviously, any $\epsilon$-independent $L^{2}(\mathbb{R})$ function or the WKB type initial data $\varphi_{\epsilon}(x)=\rho(x) e^{\frac{i S(x)}{\epsilon}}$ with $\rho(x) \in L^{2}(\mathbb{R})$ satisfy the condition of Theorem 2.1.

2) By the proof of Theorem 4.2 in [34] and Appendix $B$ of [33], we can still prove the global existence of weak solution to (2.29) with $f_{I}(x, \xi) \in \mathcal{M}^{+}\left(\mathbb{R}^{2}\right)$ and (2.24) holds only for some $\alpha>0$.

3) If the initial data $\psi(x)$ and the doping profile $b(x)$ are $C^{\infty}$ smooth, then no mollification is needed. 
Remark 2.3 The "semiclassical Vlasov-Poisson" system (cf (1.7), (1.8), (1.4)) has much better properties than the classical V-P system. This results from the fact that the "velocities" are in the bounded Brillouin zone instead of whole space. It implies that the densities $n_{m_{0}}$ ( $m_{0}$ denoting a fixed isolated energy band) inherit the $L^{p}$ natural bounds of the distribution function $f_{m_{0}}$. The existence of strong solution for this system is then easily deduced and we have moreover uniqueness of the weak solution for $f_{m_{0}, I}$ in $L^{1} \cap L^{\infty}$ as in [Ro]. As a consequence the whole sequence converges if it converges at initial time towards a bounded distribution function.

Acknowledgement. I thank Patrick Gérard for many valuable discussions.

\section{References}

[1] N.W.Ashcroft and N.D.Mermin Solid State Physics , Holt, Rinehart, 1976

[2] C. Bardos, L. Erdös, F. Golse, N.J. Mauser and H.-T. Yau, "Derivation of the Schrödinger-Poisson equation from the quantum N-particle Coulomb problem", C. R. Acad. Sci., t 334 (6), Serie I Math. (2002) 515-520

[3] C. Bardos, F. Golse and N. J. Mauser "Weak coupling limit of the $N$-particle Schrödinger equation", Math. Analysis and Applications 7 (2) (2000) 275-293

[4] C. Bardos, A. Gottlieb and F. Golse and N. J. Mauser, "Mean field dynamics of fermions and the time-dependent Hartree-Fock equation", to appear in J.d.Mathematiques Pures et Appl. (2002)

[5] C. Bardos, A. Gottlieb and F. Golse and N. J. Mauser, "Derivation of the timedependent Hartree-Fock equation : the Coulomb interaction case", manuscript

[6] P. Bechouche, N. J. Mauser and F. Poupaud, "(Semi)-nonrelativistic limits of the Dirac equation with external time-dependent electromagnetic field", Comm. Math. Phys. 197 (1998) 405-425

[7] P. Bechouche, N. J. Mauser and F. Poupaud, "Semiclassical Limit for the Schrödinger-Poisson Equation in a Crystal", Comm. Pure and Appl. Math. 54 (2001) 1-40

[8] P. Bechouche, N. J. Mauser and S. Selberg, "Nonrelativistic limit of KleinGordon Maxwell to Schrödinger-Poisson", submitted to American J. of Math. (2002)

[9] P. Bechouche, N.J. Mauser, S. Selberg, "Nonrelativistic limit of Dirac Maxwell to Schrödinger-Poisson", manuscript (2002)

[10] O. Bokanowski and N.J. Mauser, "Local approximation for the Hartree-Fock exchange potential: a deformation approach", Math.Meth. and Mod.in the Appl.Sci. 9 (6) (1999) 941-961 
[11] O. Bokanowski, B. Grébert and N.J. Mauser, "Rigorous derivation of the "Xalpha" exchange potential: a deformation approach", in "Density Functional and Ab Initio Theories Applied to Atoms, Molecules and Solids" J. Mol. Struct. (Theochem), Vol. 501-502 (2000) 47-58

[12] F. Brezzi and P.A. Markowich, "The three dimensional Wigner-Poisson problem: existence, uniqueness and approximation", Math. Meth. Appl. Sci., 14 (1991) 35-62.

[13] F. Castella, " $L^{2}-$ solutions to the Schrödinger-Poisson system: existence, uniqueness, time behavior and smoothing effects", Math. Meth. Mod. Appl. Sci., 7 (1997), 1051-1083.

[14] T. Cazenave and A. Haraux, Introduction aux problèmes d'évolution semilinéaires, Mathématiques et Applications, Ellipses, 1990.

[15] R. J. DiPerna and P. L. Lions, "Solution globales d'equations du type VlasovPoisson", C. R. Acad. Sci. Paris, 307(1988), 655-658.

[16] L. Erdös and H.-T. Yau, " Derivation of the nonlinear Schrödinger equation with Coulomb potential" Preprint (2001)

[17] P. Gérard, P. A. Markowich, N. J. Mauser and F. Poupaud, Homogenization limits and Wigner transforms, Comm. Pure Appl. Math., 50 (1997) 323-379.

[18] J. Ginibre and G. Velo, On the global Cauchy problem for some nonlinear Schrödinger equations, Ann. Inst. H. Poincaré, Analyse non linéaire, 1 (1984), 309-323.

[19] P. L. Lions, Mathematical Topics in Fluid Mechanics, Vol. 1, Incompressible Models, Oxford lecture series in Mathematics and its Applications, Oxford University Press, New York, 1996.

[20] P. L. Lions and B. Perthame, Propagation of moments and regularity for 3dimensional Vlasov-Poisson system, Invent. Math., 105(1991), 415-430

[21] P. L. Lions and T. Paul, Sur les measures de Wigner, Rev. Mat. Iberoamericana, $\mathbf{9}(1993), 553-618$

[22] A. Majda, G. Majda and Y. Zheng, "Concentrations in the one-dimensional Vlasov-Poisson equations, I : Temporal development and non-unique weak solutions in the single component case", Physica D, 74 (1994) 268-300

[23] P. A. Markowich and N. J. Mauser, The classical limit of a self-consistent quantum-Vlasov equation in 3-D, Math. Meth. Mod. Appl. Sci., 3(1993), 109124

[24] P.A. Markowich, N. J. Mauser and F. Poupaud, "A Wignerfunction Approach to (Semi)classical Limits : Electrons in a Periodic Potential", J. of Math. Phys. 35 (1994) 1066-1094 
[25] P.A. Markowich, C.A. Ringhofer and C. Schmeiser, Semiconductor Equations, Springer (1990)

[26] N. Masmoudi and N.J. Mauser, "The selfconsistent Pauli equation", Math. Monatshefte 132 (2001) 19-24

[27] N. Masmoudi, K. Nakanishi, private communication (2001)

[28] N.J. Mauser, "The Schrödinger-Poisson-Xa model", Appl. Math. Lett. 14 (2001) 759-763

[29] K. Pfaffelmoser, "Global classical solutions of the Vlasov-Poisson system in three dimensions for general initial data", J. Diff. Eq., 95 (1992) 281-303

[Ro] R. Robert, "Unicité de la solution faible à support compact de l'équation de Vlasov Poisson", C.R.A.S., Sér. I,324(8) (1997) 873-877

[30] J. Schaeffer, "Global existence of smooth solutions to the Vlasov-Poisson system in three dimensions", Comm. Partial Differential Equations, 16 (1991), 13131335

[31] E. Wigner, "On the quantum correction for thermodynamic equilibrium", Phys. Rev., 40 (1932) 742-759.

[32] A. I. Vol'pert and S. I. Hudjaev, Analysis in Classes of Discontinuous Functions and Equations of Mathematical Physics (1985)

[33] P. Zhang, Y. Zheng and N.J. Mauser, "The limit from the Schrödinger-Poisson to Vlasov-Poisson equation with general data in one dimension", Comm. Pure and Appl. Math. 55 (5) (2002) 582-632

[34] Y. Zheng and A. Majda, "Existence of global weak solutions to one-component Vlasov-Poisson and Fokker-Planck-Poisson systems in one space dimension with measures as initial data", Comm. Pure Appl. Math., 47 (1994), pp. 1365-1401

[35] Y. Zheng, "Vlasov-Poisson systems with measures as initial data", Proc of the ICIAM 95. (1995)

Wolfgang Pauli Institute C/O Inst. F. Mathematik, Univ. Wien, Strudlhofg. 4, A-1090 Wien, Austria

mauser@courant. nyu.edu

www.wpi.ac.at 